\title{
LE COORDONNANT EN TANT QU'OUTIL DE COHÉRENCE DU TEXTE : IMPLICATIONS TRADUCTOLOGIQUES
}

\begin{abstract}
Bulikowski Brunon, Le coordonnant en tant qu'outil de cohérence du texte : implications traductologiques [Co-ordinate connector as a tool for text coherence: translation implications]. Studia Romanica Posnaniensia, Adam Mickiewicz University Press, Poznań, vol. XXXVI: 2009, pp. 55-64. ISBN 978-83-232-2035-0. ISSN 0137-2475.

Co-ordinate connector as a tool for text coherence. The article shows the role of language mechanisms in respect of the co-ordination relation in the process of text coherence. The author considers the range of the phenomena studied and indicates that they go well beyond the boundaries of a complex sentence. Simultaneously, he shows their role in ensuring the isotopy of a text. In another place in the article, the author focuses his attention on the function of a co-ordinate conjunction at the inter-sentence level and, at the same time, shows the location of the phenomenon in the process of translation.
\end{abstract}

L'analyse de la coordination au niveau textuel nécessite de répondre à deux questions de base :

1. Quelle est la portée du processus : est-il limité au cadre de la phrase ou peutêtre peut-on considérer un texte entier comme une suite de phrases qui restent dans un rapport de coordination implicite ? Dans ce cas, on remettrait en cause, ce qu'on appelle en grammaire la phrase indépendante. Ce type d'hypothèse semble d'autant plus convaincante lorsqu'on analyse les textes dans lesquels la conjonction $e t$ interphrastique sert à marquer la succession des faits :

Dieu dit «Que la lumière soit ! » Et la lumière fut. / Et Dieu vit que la lumière était bonne ; et Dieu sếpara la lumière et les ténèbres. / Dieu appela la lumière Jour, et les ténèbres Nuit. Et il y eut un soir, et il y eut un matin ; ce fut le premier jour (Bible, trad. Crampon, Genèse, I, cité par Grevisse 1993).

2. Quelle est la place des procédés coordinatifs dans les mécanismes responsables de la cohérence textuelle.

Parmi les facteurs qui contribuent à la cohérence, nous allons nous concentrer sur l'isotopie du texte, qui dépend dans une large mesure de l'emploi approprié de 
reprises anaphoriques. La plupart du temps, on recourt ici à différents types d'anaphores pronominales et nominales, plus rarement, à l'anaphore adjectivale, verbale et aussi, ce qui fait l'objet de nos recherches, à l'anaphore adverbiale. Dans ce dernier cas, il faut avant tout évoquer un groupe important de connecteurs tels que en effet, ainsi, par conséquent, par contre, en revanche, qui sur le plan de la cohérence, non seulement contribuent au maintien de la continuité thématique du texte (opérations de cohésion), mais aussi jouent un rôle important dans les opérations de connexité : «l'organisation d'ensemble du texte sous la forme d'un emboîtement hiérarchique des structures propositionnelles dans les structures interpropositionnelles et de celles-ci dans le plan de texte $\gg$ (Bronckart, 1994: 55).

La nature particulière du ligament coordinatif consiste dans une sorte de condensation : il contient toujours, à des degrés divers, une liaison et un rapport. En tant que liaison, il est assez comparable à un pronom : comme lui il représente le tout ou une partie du terme ou du membre qu'il relie au terme suivant. En tant qu'outil de rapport, il ajoute à cette liaison une détermination : il possède donc en réalité une double fonction de sens (Bally 1965: 67-69 ; Antoine 1996: 330). Dans la phrase

Il pleut; aussi nous ne sortirons pas,

aussi $=1$ ) le fait qu' «il pleut »

entraîne :

2) dans ces conditions (ou : pour cette raison).

Pour expliquer la signification du connecteur dans cette phrase, Bally propose un schéma qui consiste à répartir les deux coordonnées entre deux interlocuteurs ; on obtiendra alors :

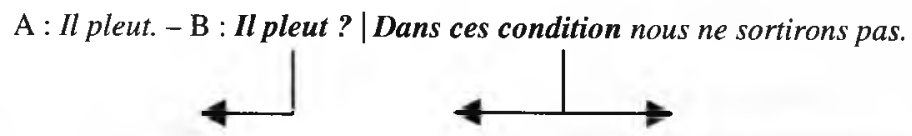

L'outil coordonnant garde ainsi une attache avec le premier terme (membre), tandis que le sens déterminatif, exprimant la modalité de la relation coordinative, l'oriente en même temps vers le second (Antoine, op. cit.: 339).

Si l'on compare les conjonctions et les adverbes qui remplissent le rôle d'outils coordinatifs, on constatera que, du point de vue de la fonction déterminative, il y a de la conjonction à l'adverbe une progression croissante. La fonction déterminative est une source d'impuretés pour la coordination et la rapproche de la liaison de subordination (op. cit.: 352 ) :

Il pleut; mais nous sortons,

Il pleut ; cependant nous sortons,

Il pleut ; malgré cela nous sortons. 
Par contre, la fonction de représentation diminue à mesure que se développe le facteur déterminatif. Dans le dernier exemple, les deux rôles - représentatif et déterminatif - sont répartis entre deux mots : cela pour le premier, malgré pour le second ; la coordination grammaticale explicite n'existe pour ainsi dire pas : les deux «phrases » sont logiquement enchaînées, mais formellement indépendantes (ibid.).

Quant à la fonction de représentation, toute conjonction de coordination renferme un pronom (Bally, op. cit.: 68). Pour illustrer le problème, Gérald Antoine rapproche, sur le plan psychologique, les coordinations suivantes :

Vous dormez : par ce fait même vous oubliez vos ennuis, et

Vous dormez, et par le fait même vous oubliez vos ennuis.

Selon lui, si nous disions :

Vous dormez et par ce fait mềme vous oubliez vos ennuis,

nous créerions un effet d'insistance en doublant le facteur représentatif. Et si, au contraire, nous disons :

Vous dormez : par le fait même vous oubliez vos ennuis.

il se produirait une certaine absence de liaison. Toutefois, le sujet parlant utiliserait alors une modification de ton très sensible, la voix s'élevant bien davantage à la fin du premier membre pour redescendre ensuite. C'est donc l'intonation qui aurait pour but d'exercer dans ce dernier cas la fonction représentative (Antoine, op. cit.: 344-345).

Bien que les conjonctions de coordination et les adverbes coordinatifs diffèrent par la proportion entre la fonction représentative et la fonction déterminative, Albert Sechehaye (1950: 166) identifie ces derniers aux conjonctions :

Ceux de ces « adverbes » qui expriment l'enchaînement logique des idées méritent, pour autant qu'ils sont bien spécialisés dans ce rôle, le nom de conjonctions. Les conjonctions de coordination ne sont pas autre chose. Généralement on réserve ce nom à des termes d'un caractère tout à fait particulier et qui lient des propositions intimement unies dans une même phrase. En français, par exemple, on met généralement sous cette rubrique les particules $e t, o u, n i$, mais, donc, or et car. Les termes que nous avons énumérés plus haut [au contraire, $d u$ moins, par consé-quent, cependant, alors, etc.] quoique marquant une liaison moins étroite, ne peu-vent pas en être séparés.

Selon Sechehaye, les expressions ci-dessus sont le résultat du procédé au cours duquel un elément se détache psychologiquement de l'agencement logique de la phrase et prend vis-à-vis du reste une valeur de prédicat ou de sujet psychologique. Il illustre ce phénomène à l'aide de la phrase :

Je n'ai jamais dit cela à personne, 
qui peut appuyer sur jamais, sur cela, sur personne, ou même sur le verbe dire selon que la protestation porte plus spécialement sur tel ou tel point (Sechehaye, op. cit.: 127). Cette importance qu'un terme prend relativement aux autres dans le prédicat ou dans le sujet peut aller jusqu'à troubler le rapport psychologique qui devrait exister selon les normes de la grammaire entre les deux parties de la phrase, comme c'est le cas dans

Jamais je ne l'avais vu si en colere,

ou l'on emploiera un fort accent prédicatif sur jamais. Nous avons dans cet exemple un élément qui, détaché de son agencement logique, se place devant l'ensemble et devient le vrai prédicat psychologique (rhème) par rapport au reste qui a la qualité d'un sujet (thème) postposé. Au contraire, dans la phrase :

Avec cet argent j'ai acheté une chaîne de montre,

les premiers mots, détachés eux aussi de leur ensemble logique, peuvent avoir une valeur purement introductive et jouer vis-à-vis du reste le rôle d'un véritable sujet. Ce dernier cas est le plus fréquent (op. cit.: 127-128).

Il y a toutes sortes de termes qui peuvent être détachés de la manière décrite ci-dessus : ils sont très divers tant au point de vue du sens qu'au point de vue grammatical. Sechehaye propose de les désigner sous le terme commun de compléments de proposition, premièrement, parce qu'ils apportent une idée qui peut s'agencer logiquement avec celle qu'exprime le reste de la phrase, et deuxièmement, parce que ce reste de la phrase représente en général l'équivalent d'une proposition complète et se suffisant à elle-même. Cependant le rapport psychologique qui s'établit entre le complément de proposition et le reste de la phrase n'est pas celui d'un élément principal à son complément : «ce sont deux énoncés successifs dont l'un introduit ou complète l'autre ; il s'agit d'un énoncé en deux mouvements » (op. cit.: 164-165).

Le complément de phrase fait aussi l'objet d'études de Pierre Le Goffic (1993: 13) qui essaie de le situer par rapport aux structurations logique et fonctionnelle de la phrase, comme dans le tableau suivant:

Tableau 1

\begin{tabular}{|c|c|c|c|c|}
\hline sujet & \multicolumn{3}{|c|}{ prédicat } & éléments extra-prédicatifs \\
\hline sujet & verbe & $\begin{array}{c}\text { compléments } \\
\text { essentiels }\end{array}$ & $\begin{array}{c}\text { compléments accessoires } \\
\text { intra-prèdicatifs }\end{array}$ & $\begin{array}{c}\text { compléments accessoires } \\
\text { extra-prédicatifs }\end{array}$ \\
\hline
\end{tabular}

Il résulte du schéma ci-dessus que, suivant leur portée, les compléments accessoires se laissent répartir en éléments intra-prédicatifs et extra-prédicatifs. Comme la présente étude concerne essentiellement les propriétés du ligament coordinatif à l'échelon textuel, dans la suite de notre travail, les notions de compléments intraprédicatif et extra-prédicatif seront restreintes à celles de circonstants intra-prédicatif et extra-prédicatif. 
En ce qui concerne le circonstant intra-prédicatif (ou circonstant de prédicat), il est rattaché au verbe et fait partie du prédicat, qu'il spécifie sous un (ou plusieurs) rapport(s). Le circonstant extra-prédicatif (ou circonstant de phrase), quant à lui, n'appartient pas au prédicat, mais porte sur la phrase dans son ensemble ; on considère qu'il porte sur la relation entre le procès exprimé par la phrase et son énonciation, ou entre ce procès et l'état du monde (op. cit.: 457-458). Comparons les exemples suivants, cités par Le Goffic (pp. 82 et 484) :

A-t-il parlé franchement ? "sa parole a-t-elle, oui ou non, été franche ?"

Franchement. a-t-il parlé ? "je vous demande franchement si, oui ou non, il a parlé"

Je ne t'oublierai jamais. "l'oubli ne se produira à aucun moment"

Jamais je ne t'oublierai. "jamais il ne sera vrai que je t'oublierai"

L'extériorité des circonstants extra-prédicatifs se manifeste normalement par la multiplicité des places qu'ils peuvent occuper, en construction détachée (avec de subtiles variations de portée et d'effets de sens)

(Heureusement,) les secours (, heureusement,) sont (, heureusement,) arrivés

(, heureusement,) à temps (, heureusement) (op. cit.: 459).

Dans notre étude, nous nous concentrons avant tout sur la position initiale étant donné sa spécificité et son rôle dans la construction d'un texte. Selon Le Goffic c'est l'endroit par excellence pour énoncer des éléments extra-prédicatifs : «En début d'énoncé, l'énonciateur dispose d'une zone de liberté relative, avant d'être pris dans le réseau serré des relations syntaxiques de son énoncé (réseau dont il peut toujours, certes, sortir par la construction détachée, mais d'une façon plus coûteuse). [...] Le début de phrase est le lieu privilégié pour les liens avec la phrase précédente (d'où des éléments anaphoriques fréquents), les indications thématiques, les indications concernant l'organisation du discours, et autres instructions à l'usage du récepteur » (op. cit.: 85).

Les circonstant initiaux se laissent répartir, par ordre d'extériorité décroissante, en quatre groupes (op. cit.: 460-466) :

- portant sur l'énonciation : A propos, savez-vous qui j'ai rencontré !

- portant sur l'énoncé comme un tout: Heureusement, il n'est pas tombé !

- donnant un cadre à la phrase : La nuit, tous les chats sont gris.

- indiquant une "manière de phrase" : Brusquement, Paul est parti.

C'est dans le premier des quatre groupes ci-dessus, le plus extérieur par rapport au prédicat, celui renvoyant à la situation d'énonciation et portant sur certains aspects de l'énonciation même de la phrase, que l'auteur range les éléments qui permettent l'organisation du discours :

et, mais, donc, par conséquent, en revanche, en effet...

Il met ainsi sous une même étiquette tant les adverbes anaphoriques dont parle A. Sechehaye que les expressions traditionnellement désignées sous le nom de conjonctions. 
Comme nous l'avons vu au début de cette étude, dans une citation biblique, la conjonction de coordination, dont les valeurs anaphorique et extra-prédicative ont été indiquées plus haut, peut être employée, contrairement à l'usage ordinaire, en position inter-phrastique. Il s'agit d'un outil linguistique dont la fréquence d'emploi varie sensiblement d'un auteur à l'autre. Parmi les œuvres littéraires françaises qui abondent en ce type de construction, il faut certainement ranger Le Petit Prince d'Antoine de Saint-Exupéry, œuvre philosophique et poétique qui, sous l'apparence d'un conte pour enfants, aborde des thèmes profonds comme la vie, l'amour, l'amitié.

Il suffit d'analyser la partie narrative du texte, qui suivant l'édition, compte de 70 à 100 pages, pour constater que l'emploi de la conjonction de coordination interphrastique est une construction importante du point de vue quantitatif :

Tableau 2

\begin{tabular}{|c|c|}
\hline $\begin{array}{c}\text { Conjonction de coordination inter-phrastique dans la narration du } \\
\text { Petit Prince d'A. de Saint-Exupéry }\end{array}$ \\
\hline Et... & 84 \\
\hline Car... & 6 \\
\hline Mais... & 54 \\
\hline Total & 144 \\
\hline
\end{tabular}

Qu'il s'agisse d'un phénomène non négligeable, on peut aussi s'en convaincre lorsqu'on rapproche le texte original de sa traduction en anglais, The Little Prince, faite par Irène Testot-Ferry (1995). Nous pouvons alors constater que le plus souvent la traductrice emploie une même construction syntaxique, ce qu'on peut illustrer avec les chiffres suivants :

Tableau 3

\begin{tabular}{|c|c|}
\hline \multicolumn{2}{|c|}{ The Little Prince (trad. I. Testot-Ferry, 1995) } \\
\hline Et... $\rightarrow$ And... & $75(89 \%)$ \\
\hline Car... $\rightarrow$ For... & $6(100 \%)$ \\
\hline Mais... $\rightarrow$ But... & $46(85 \%)$ \\
\hline Total & $127(88 \%)$ \\
\hline
\end{tabular}

"Tu viens donc d'une planète?"

Mais il ne me répondit pas. Il hochait la tête doucement tout en regardant mon avion :

"C'est vrai que, là-dessus, tu ne peux pas venir de bien loin..." $\underline{\text { Et }}$ il s'enfonça dans une rêverie qui dura longtemps.

'So you've come from another planet?'

But he didn't reply. He nodded gently whilst gazing at my aeroplane.

'It is true that on that you can't have come from very far away...'

And he sank into a long reverie. 
Il faut ajouter ici que la traductrice qui a réduit le nombre de conjonctions de coordination en position inter-phrastique d'environ $10 \%$ lors du processus de traduction, en a introduit à son tour une dizaine dans d'autres passages du texte sans qu'on ne puisse, quelquefois, leur trouver un équivalent explicite dans la version française :

J'avais ainsi appris une seconde chose très importante: c'est que sa planète d'origine était a peine plus grande qu'une maison.

Ça ne pouvait pas m'étonner beaucoup.

Thus I had learned a second very important thing. That his planet of origin was scarcely larger than a house.

But that did not really surprise me very much.

L'analyse commentée plus haut pourrait suggérer une hypothèse selon laquelle l'emploi de la conjonction inter-phrastique serait caractéristique du type particulier de discours que constituent les textes pour enfants ou ceux qui sont rédigés dans un climat de textes pour enfants. En effet, la construction en question permet d'éviter des phrases trop longues, surchargées d'informations et rend par là même le texte écrit moins lourd et plus assimilable.

Toutefois parmi les œuvres littéraires les plus connues du genre susmentionné, il y en a où le procédé décrit n'apparaît que sous forme de cas isolés. Il en est ainsi, par exemple, pour les contes de Charles Perrault. La Belle au bois dormant, Le Petit Chaperon rouge, Le Maître chat ou le Chat botté et Cendrillon ou la petite pantoufle de verre, titres les plus connus, constituent ensemble un texte d'environ 20 pages, qui ne comporte pour autant que 3 conjonctions inter-phrastiques, tandis que dans le texte de Saint-Exupéry, sur le même nombre de pages, on a pu en repérer, en moyenne, plus de 20 exemples. Cependant, il est très intéressant d'analyser les contes de Perrault dans la perspective de la traduction en d'autres langues. Si dans le cas du Petit Prince on a noté un certain équilibre entre la version française et anglaise en ce qui concerne l'emploi des conjonctions inter-phrastiques Et/And, Car/For et Mais/But, respectivement 144 et 140 (partie narrative),

Tableau 4

\begin{tabular}{|l|c|}
\hline \multicolumn{2}{|c|}{ Conjonctions de coordination inter-phrastiques } \\
Et/And, Car/For et Mais/But \\
dans la narration du Petit Prince \\
\hline Le Petit Prince & 144 \\
\hline The Little Prince & 140 \\
\hline
\end{tabular}

les traductions des contes de Perrault, non seulement en anglais (Philip, Simborowski, 1993) mais aussi en polonais (Januszewska, 2004), comportent un nombre sensiblement plus élevé des constructions étudiées par rapport à la version originale : 
Tableau 5

\begin{tabular}{|l|c|}
\hline \multicolumn{2}{|c|}{ Conjonctions de coordination inter-phrastiques } \\
Et / And /I, A \\
\multicolumn{2}{|c|}{ dais / But /Ale, Lecz la narration des contes de Ch. Perrault } \\
\hline $\begin{array}{l}\text { La Belle au bois dormant } \\
\text { Le Petit Chaperon rouge }\end{array}$ \\
$\begin{array}{l}\text { Le Maître chat ou le Chat bottê } \\
\text { Cendrillon ou la petite pantoufle de verre }\end{array}$ \\
\hline $\begin{array}{l}\text { The Sleeping Beauty } \\
\text { Little Red Riding Hood }\end{array}$ \\
$\begin{array}{l}\text { Puss-in-Boots } \\
\text { Cinderella }\end{array}$ \\
\hline $\begin{array}{l}\text { Spiaca królewna } \\
\text { Maty Czerwony Kapturek } \\
\text { Imć kot, czyli kot w butach } \\
\text { Kopciuszek, czyli pantofelek z popieliczki }\end{array}$ \\
\hline
\end{tabular}

Il faut ici souligner que dans le cas de plus de la moitié des exemples dans la version polonaise, la construction étudiée n'a pas son équivalent explicite dans les deux autres versions. Un quart des conjonctions inter-phrastiques polonaises, par contre, ont leurs correspondants intra-phrastiques tant en français qu'en anglais :

Każda panna na takie pogaduszki utożylaby im wtosy na bakier. Ale Kopciuszek miat dobre serce $i$ uczesat siostry znakomicie.

Une autre que Cendrillon les aurait coiffées de travers; mais elle était bonne, et elle les coiffa parfaitement bien.

Anyone else but Cinderella would have tangled their hair, but she was good, and she styled it to perfection.

Il est aussi intéressant d'ajouter que dans deux passages des textes examinés, tant dans la version anglaise que polonaise, les traducteurs ont introduit la conjonction inter-phrastique parallèlement :

- Impossible? reprit l'Ogre, vous allez voir ", et en même temps il se changea en une Souris, qui se mit à courir sur le plancher. (Le Maître chat...)

"Impossible?" said the ogre. "You'll see." And as he spoke he changed into a mouse, which began to run about on the floor.

- Trudno asanowi uwierzyć! - wpadt na niego wilkotak. - Zaraz się przekonasz!

I w tejże chwili przemienit się w mysz, która puściła się biegiem po podtodze.

La première conclusion qui vient à l'esprit lors de l'analyse des textes cités dans cette étude, c'est que l'emploi de la conjonction de coordination inter- 
phrastique est un procédé syntaxique et stylistique qu'on ne peut certainement pas qualifier de recherché et qu'on rencontre dans différents systèmes linguistiques, encore qu'il soit employé par les auteurs avec une fréquence très variée. De plus, en tant qu'articulation du texte, la conjonction susdite, laisse des traces durables dans le processus de traduction, ces derniers contribuant sensiblement à l'isotopie du texte traduit. Souvent, il s'agit ici d'une construction analogue dans la langue cible, et dans la plupart des autres cas, d'une autre expression porteuse du sens anaphorique, que ce soit une conjonction intra-phrastique ou une autre catégorie grammaticale.

C'est aussi sur le plan de la traduction qu'un autre aspect de la construction en question se fait voir d'une manière plus nette : il s'agit des cas où la conjonction, dont la propriété essentielle est de lier deux séquences à l'intérieur d'une phrase complexe, au niveau intra-phrastique, est rendue dans le texte traduit par sa variante inter-phrastique, ou à l'inverse, ce qui permet de mieux distinguer cette «propriété de lier » mais sur l'échelon textuel et par là même son rôle dans la cohérence du texte. Si nous ajoutons aux observations présentées ci-dessus le fait que le phénomène décrit offre, dans certains cas, la possibilité d'éviter des constructions phrastiques trop longues qui pourraient troubler la lisibilité du texte, il ne sera pas douteux que nous avons affaire à un outil linguistiques qui peut faciliter la rédaction d'un écrit et sur lequel la grammaire de texte devrait attirer l'attention lors du processus didactique.

Nous avons vu, dans la première partie de cette étude, que parmi les éléments qui se détachent de la structure ferme sujet-prédicat et qui se mettent en relation avec le reste du texte dont la phrase fait partie, on peut distinguer aussi d'autres connecteurs coordinatifs : il s'agit des nombreux adverbes qui, de même que la conjonction de coordination, non seulement assument une fonction déterminative en spécifiant le caractère de la ligature, mais aussi ont la propriété d'assurer la continuité thématique grâce à leur valeur anaphorique. Étant donné que les connecteurs en question assument les deux fonctions tant à l'échelon intra-phrastique qu'interphrastique, on peut supposer que la relation, qualifiée souvent de coordination, dépasse le cadre d'une phrase complexe et peut s'étendre sur une séquence de texte plus longue. De l'autre côté, la présence du facteur déterminatif dans le sémantisme des ligaments coordinatifs, même ceux qui explicitent la relation la plus proche de la coordination pure, peut remettre en question la notion de phrase indépendante, du moins dans le cadre d'un texte cohérent. Quoi qu'il en soit, il résulte de ce qui précède, que le rôle des coordonnants dans l'organisation du texte n'est pas négligeable et que leurs sélection et emploi appropriés constituent un facteur important responsable de la cohérence et de la qualité textuelle. 


\section{BIBLIOGRAPHIE}

Antoine G. (1996), La coordination en français, Paris : Éditions d'Artrey.

Bally Ch. (1965), Linguistique générale et linguistique française, $4^{\mathrm{e}}$ édition revue et corrigée, Berne : Éditions Francke.

Bronckart J.-P. (1994), Le fonctionnement des discours. Un modèle psychologique et une méthode d'analyse, Lausanne-Paris : Delachaux \& Niestlé.

Carter-Thomas S. (2000), La cohérence textuelle. Pour une nouvelle pédagogie de l'écrit, ParisMontreal : L'Harmattan.

Grevisse M., 1993, Le bon usage. Grammaire française, Paris-Louvain-la-Neuve : Duculot, $\left(13^{\mathrm{e}}\right.$ édition refondue par A. Goosse).

Le Goffic P. (1993), Grammaire de la Phrase Française, Paris : Hachette.

Reichler-Béguelin M.-J., Denervaud M., Jespersen J. (1990), Écrire en français. Cohèsion textuelle et apprentissage de l'expression écrite, Neuchâtel-Paris : Delachaux et Niestlé.

Riegel M.. Pellat J.-C., Rioul R. (1998), Grammaire méthodique du français, Paris : PUF.

Sechehaye A. (1950), Essai sur la structure logique de la phrase, Paris : Librairie Ancienne H. Champion, Éditeur É. Champion.

\section{CORPUS}

Perrault Ch. (1993), The complete Fairy Tales of Charles Perrault, transl. by Philip N., Simborowski N., with an introduction and notes on the stories by Philip Neil, New York : Clarion Books.

Perrault Ch. (2002), Contes, Paris : L'Aventurine.

Perrault Ch. (2004), Bajki, trad. H. Januszewska, Wrocław : Siedmioróg.

Saint Exupéry A. de (1995), The Little Prince, transl. by I. Testot-Ferry, Ware : Wordsworth Editions Ltd.

Saint Exupéry A. de (2000), Le Petit Prince, avec des aquarelles de l'auteur, Paris : Gallimard. 